\title{
Acquisition and Analysis of a 1.2 Gbyte FIB/SEM Tomographic Spectral Image
}

\author{
Paul G. Kotula \\ Sandia National Laboratories, PO Box 5800, Albuquerque, NM 87185-0886
}

Spectral imaging with $\mathrm{x}$-rays, where a complete $\mathrm{x}$-ray spectrum is acquired from each point in an array of points, is a powerful microanalytical approach. Combined with multivariate statistical analysis (MSA), this allows us to efficiently mine the very large amounts of data in spectral images and quickly extract the chemically relevant components [1-4]. Spectral imaging can be made even more powerful by correlating multiple spectral images and analyzing them simultaneously. These can be a simple montage covering a larger area than possible with a single image or sampling volume elements instead of areal elements. This latter technique, called tomographic spectral imaging, provides comprehensive 3D chemical information about the specimen [3]. The serial sectioning or direct tomographic methods include sectioning with focused ion beams [3] as well as metallographic sectioning, followed by the serial collection of $x$-ray spectral images. The challenge with this approach is that the data sets can exceed many gigabytes in size.

Figure 1 shows the results of the analysis of a tomographic spectral image collected by serial FIB sections of corroded sample followed by SEM-EDS spectral imaging. The entire data set consisted of 25 total sections, each consisting of 256 by 192 pixels by 512 channels ( $0-5 \mathrm{kV}$ x-ray spectra). A data set of this size has over 1.2 million individual X-ray spectra (voxels) and over six-hundred million data elements. The data set was 1.2 Gbytes in size and took 2 minutes ( $\sim 600$ Mbytes/minute) to analyze with Sandia's Automated eXpert Spectral Image Analysis MSA software running on a dual dual-core Xeon-processor PC workstation running Microsoft's XP-x64 operating system and equipped with 8 Gbytes of RAM. The sample consists of a free-machining brass substrate (brass with $\mathrm{Pb}$ particles) which has been electroplated with several microns of $\mathrm{Cu}$ and then $1 \mathrm{~mm}$ each of $\mathrm{Ni}$ and $\mathrm{Au}$. It was then subjected to a standard industrial corrosive environment. The corroded regions were readily identified on the surface in the SEM and then tomographic spectral images were acquired as previously described [3]. Multivariate statistical analysis (MSA) was performed so as to maximize the mutual spatial simplicity of the component images [2,4]. The MSA output consisted of six components which are rendered in Fig. 1. The component images were rendered in 3D with the Imaris software package from Bitplane AG. Fig. 2 shows a 3D component image overlay without the $\mathrm{Au}$ film and $\mathrm{Ni}$ film components rendered. It is clear that the corrosion process has moved $\mathrm{a} \mathrm{Pb}$ particle up to the near-surface. The root cause for the localized corrosion in this case was the presence of a scratch in the protective Au coating which allowed corrosive attack of the underlying materials. Microanalysis, serial sectioning, and MSA provide a comprehensive 3D solution to this very large analytical problem.

[1] P.G. Kotula, M.R. Keenan and J.R. Michael. Microsc. Microanal. (2003) 9 1-17. 
[2] M.R. Keenan and P.G. Kotula, Microsc. Microanal. (2004) 10 [Suppl. 2 Proc] 874CD. [3] P.G. Kotula, M.R. Keenan and J.R. Michael. Microsc. Microanal. (2006) 12 36-48. [4] M.R. Keenan, Surf. Int. Anal. (2008) DOI 10.1002/sia.2949

The authors would like to thank Garry Bryant and Michael Rye at Sandia for FIB serial sectioning and spectral image acquisition. Sandia is a multiprogram laboratory operated by Sandia Corporation, a Lockheed Martin Company, for the United Stated Department of Energy's (DOE) National Nuclear Security Administration (NNSA) under contract DE-AC0494AL85000.

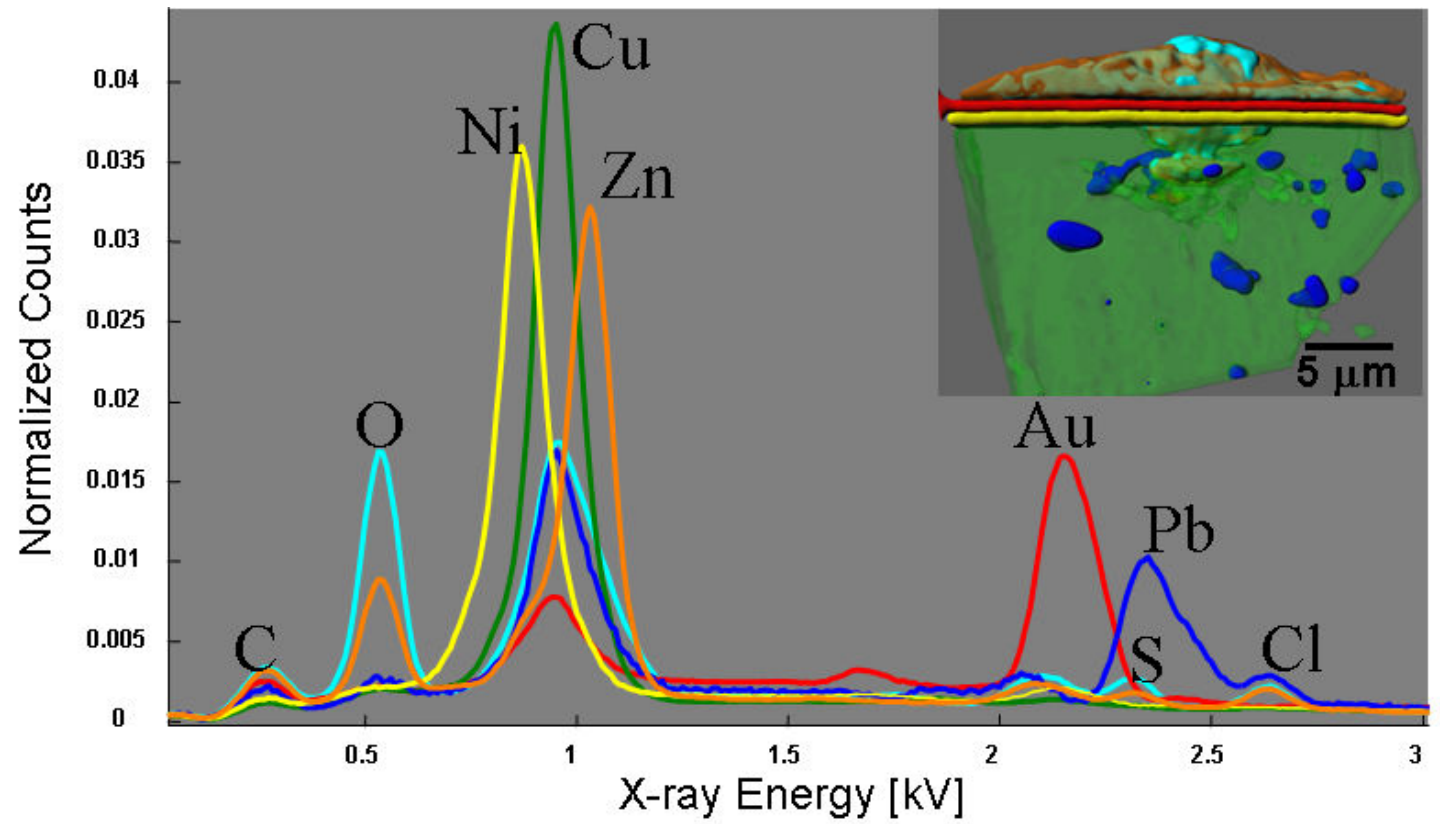

Figure 1. Results from FIB sectioning, $\mathrm{X}$-ray spectral image acquisition and multivariate statistical analysis of a corrosion sample. Red $=\mathrm{Au}$ film, Green (translucent) $=\mathrm{Cu}$, Blue $=\mathrm{Pb}, \mathrm{Cyan}=\mathrm{Cu}-\mathrm{Zn}-\mathrm{S}-\mathrm{Cl}-\mathrm{O}$ corrosion product, Yellow $=\mathrm{Ni}$ film, and Orange $($ translucent $)=\mathrm{Zn}-\mathrm{Cl}-\mathrm{S}-\mathrm{O}$ corrosion product .

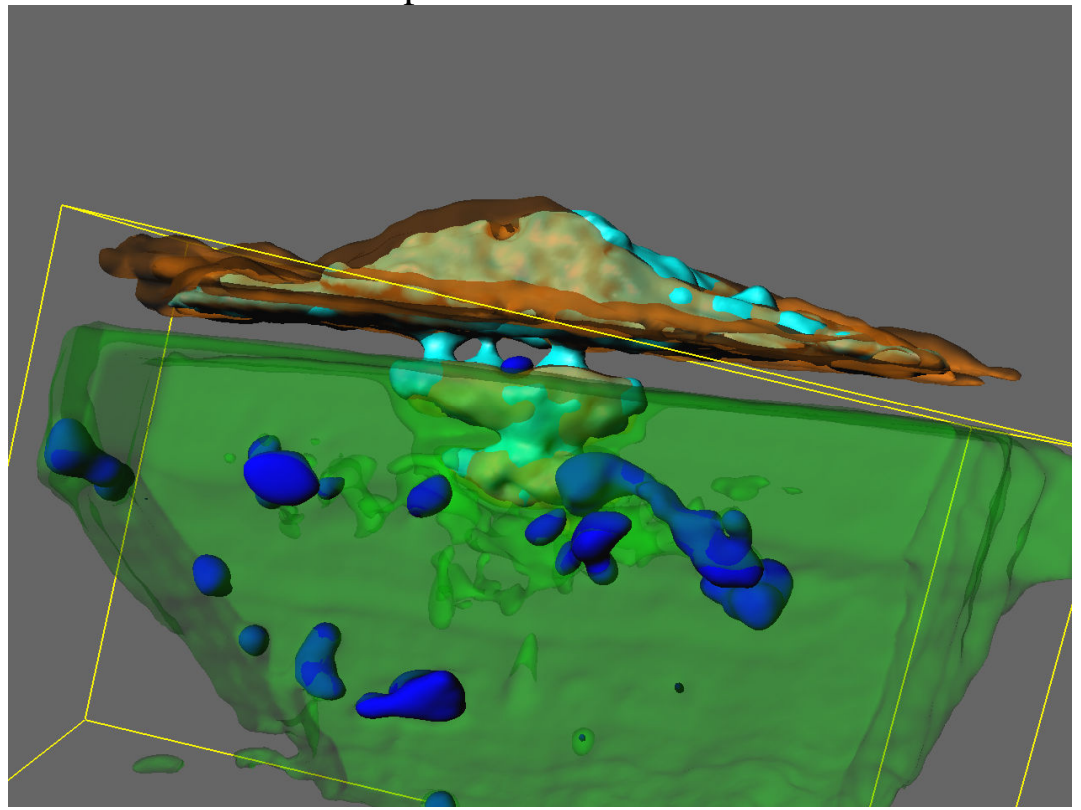

Figure 2. 3D component image overlay without $\mathrm{Au}$ and Ni films rendered. 